\title{
Entre cuerpos humanos y plegados: Ejemplos de afecto y cuidado en Bogotá
}

\section{Between human and folded bodies: Examples of affection and care in Bogotá}

Gerardo Gacharná Ramírez ${ }^{1}$

DOI: 10.29151/hojasyhablas.n20a9

\section{Resumen}

El objetivo del ejercicio fue comprender las relaciones afectivas entre plegados ${ }^{2}$ de origami que han sido cuidados por un tiempo significativo y las personas que los cuidan. La vida material de los objetos, desde el posthumanismo, sirvió como aparato teórico, y el investigar con objetos como marco metodológico, seleccionando en Bogotá cuatro parejas de plegados y sus cuidadoras ${ }^{3}$ para registros fotográficos y entrevistas filmadas en los lugares donde habitaban los plegados. Como un componente reflexivo, el autor y su plegado también participaron como pareja. En cuanto al cierre, algunas formas de relación plegado-cuidadora (paternidades, pactos, perdones, retornos e in-materialidades) fueron presentadas a partir del ejercicio, junto a tres propuestas para futura indagación: conocer otras posibles configuraciones entre cuerpos humanos y plegados, estudiar en mayor profundidad las formas de relacionarse entre origamistas y nuevamente los plegados, y estudiar de forma material el vínculo entre creadora, primer plegado y el llamado "modelo" en el origami.

Palabras clave: Origami; objetos; materialidad; cuerpos; afecto; cuidado.

\begin{abstract}
The objective of the exercise was to understand the affective relationships between origami folds that have been taken care by participants for a significant amount of time. From a post humanism perspective, the material life of objects served as the theoretical approach; and the research with objects as the methodological framework, selecting in Bogotá four pairs of folds and their caretakers for photographic records and filmed interviews in the places where the folds are. As a reflective component, the author and his fold also participated as a pair. Regarding the finishing statement, some forms of the fold-caretaker relationship (paternities, pacts, forgiveness, returns, and immaterialities) were presented based on the exercise, along with three proposals for future inquiry: to get to know other possible configurations between human and folded bodies, to study in greater depth the forms of relating between origamists and folds, and to study materially the link between creator, first fold, and what is called a "model" in origami.
\end{abstract}

Keywords: Origami; objects; materiality; bodies; affection; care.

\footnotetext{
${ }^{1}$ Estudiante del Doctorado de Ciencias Humanas y Sociales, Universidad Nacional de Colombia. Correo: ggacharna@unal.edu.co

${ }^{2}$ Yo empleo en todo el artículo el término "plegado" para referirme al objeto material hecho a través de la técnica del origami y "modelo" para denotar al diseño abstracto que puede ser plegado de forma repetida y por distintas personas.

${ }^{3}$ En el texto sigo el ejemplo de Rey y Boesel (2014) de emplear la forma femenina de ciertas palabras para incluir todo género; esto con la intención de "contrarrestar la tradición más antigua de usar pronombres masculinos generalizados” (p. 186, la traducción es mía) y, a la vez, procurar una mayor legibilidad que al cambiar una vocal por “x” o “@”.
} 


\section{Introducción}

Los plegados de origami más antiguos en existencia son parte de la colección del Museo Metropolitano de Arte (s.f.), en Nueva York. Se trata de una serie de plegados formales para caligrafía, hechos por una discípula de una de las maestras de la escuela Ogasawara de Japón; serie que data de 1697

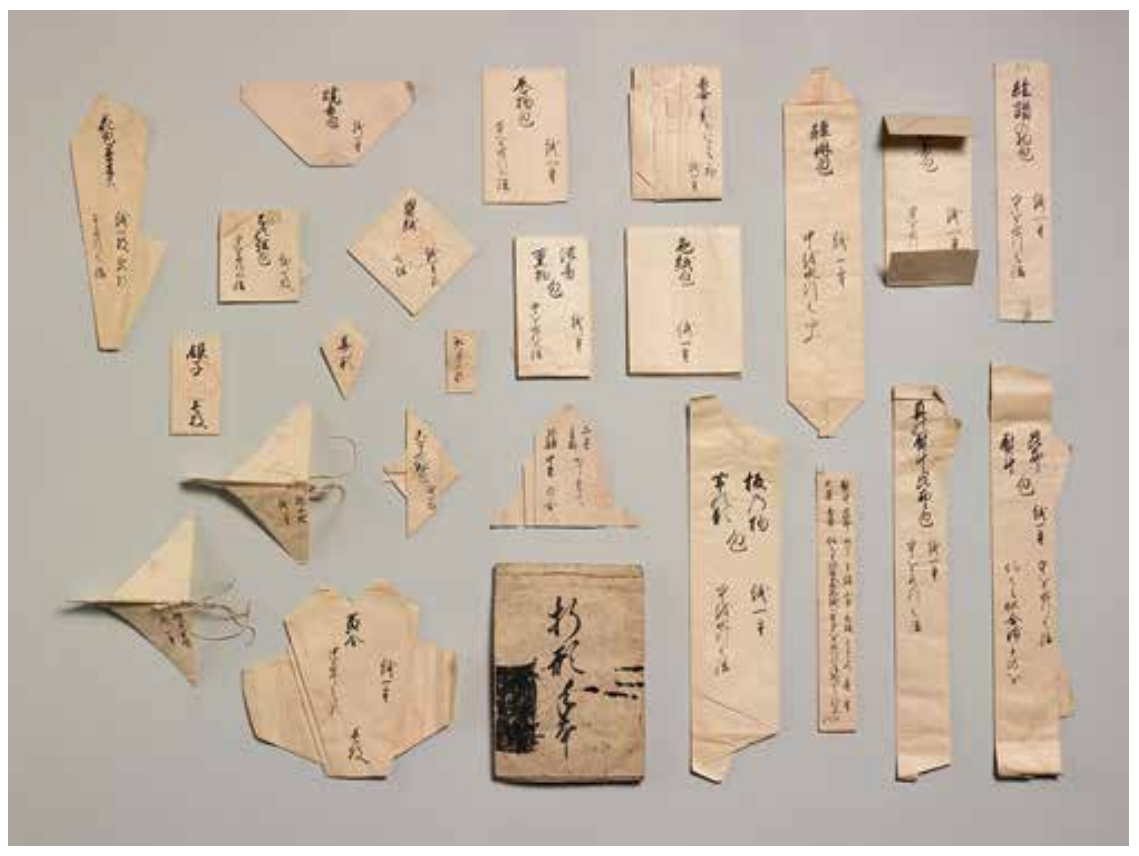

Figura 1. Muestra parcial de los plegados de 1697 de la colección del Museo Metropolitano de Arte.

¿Qué lleva a que un plegado de origami se convierta en un objeto de cuidado y, por ejemplo, en el caso citado haya sido conservado por más de trescientos años? En la actualidad, esa no parece ser la práctica más común con los plegados de origami. Lang (1987), uno de los origamistas más reconocidos en las últimas décadas, manifestó en una ocasión: "la mayoría de personas que pliegan, aceptan que el origami es plegado en un momento y convertido en una bola de papel para tirar, al siguiente" (Traducción propia, p. 21). Algunas personas han relacionado este carácter efímero del origami con una cuestión espiritual. Por ejemplo, Romero Merizalde (2015) afirmó que las budistas practicaban el plegado de papel, relacionándolo con el ejercicio de desprenderse del plegado luego de terminarlo y, también, desprenderse del apego afectivo entre ambas. El autor continúa citando las escrituras sagradas del budismo, el Dhamma- pada, según las cuales el apego conlleva al dolor y al sufrimiento, por lo cual nos liberamos de ambos males al alejarnos de los apegos.

Yo, por el contrario, me posiciono en defensa de los apegos afectivos entre cuerpos humanos y plegados. Tales apegos forman parte de nuestra realidad corpórea/material, reafirman nuestra presencia y existencias entrelazadas. $\mathrm{O}$ como lo manifestaron Callén Moreu y López Gómez (2019), por el bien del planeta, necesitamos apegarnos más a las cosas, no desprendernos de ellas. Desde esta postura, yo me pregunté por el cuidado de unos plegados de origami por parte de ciertas personas, específicamente habitantes de Bogotá, y su relación con el afecto. Con "cuidado" me refiero a una forma de relación en la cual, a través de esfuerzo y acciones concretas, se procura el bienestar de algo-alguien. De modo que, 
el objetivo del presente ejercicio fue comprender las relaciones afectivas entre plegados de origami que han sido cuidados por un tiempo significativo y las personas que los cuidan.

Empezaré ofreciendo una idea general sobre el origami, aclaraciones respecto a algunas creencias populares, y su surgimiento tanto en el ámbito mundial como local. Luego presentaré el aparato teórico del ejercicio de indagación: la vida material de los objetos, centrándome particularmente en su conexión con el afecto. Después de eso, ofreceré detalles del método que apropié, desde el investigar con objetos. De ahí, pasaré a describir y analizar las cinco relaciones entre cuerpos plegados y cuerpos humanos del ejercicio, y finalmente, ofreceré algunas ideas como cierre de todo el proceso.

\section{Brochazo histórico}

Joseph Wu definió al origami como "una forma de arte del papel en el que el plegado es la técnica principal para lograr un efecto" (citado por Lister, s.f. c, párr. 1). Tomamos una hoja y, a partir de una serie de dobleces, logramos una forma deseada. Popularmente, se tiene la creencia que su origen es inequívocamente japonés. Sin embargo, hay quienes declaran que también se desarrolló de forma independiente en otros lugares del planeta (Hatori, s.f.; Lister, 2003; Lang, 2014).

Hatori (s.f.) cita al libro de origami más antiguo que se conoce, Tsutsumi-no Ki por Ise Sadatake de 1764 para declarar que, en Japón, el origami empezó en la era Muromachi (1336 - 1557). Se trataba de plegados de papel de carácter ceremonial que eran transmitidos a la clase samurái: plegados que acompañaban a los obsequios con la intención de desear buena fortuna (noshi), y una mariposa hembra (Mecho) y otra macho (Ocho) para decorar las botellas de sake en las bodas sintoístas (Origami Resource Center, s.f.). Por su parte, la evidencia más antigua de la conocida grulla (Orizuru) y otros modelos figurativos tradicio- nales del país nipón corresponden al siglo XVIII (Hatori, s.f.). Aunque Lister (s.f. a) manifiesta que la grulla ya era popular en Japón a finales del siglo XVI.

Pasando a Europa, parece haber evidencia de una caja hecha a partir de cortes y pliegues en la primera mitad del siglo XV (da Riva, s.f.), del tradicional barco de papel a finales del mismo siglo y posible evidencia del modelo actualmente conocido como la bomba de agua, de inicios del siglo XVII (Hatori, s.f.). Aparte de eso, en la zona de Bavaria y países aledaños, durante los siglos XVI, XVII y XVIII, los llamados "certificados bautismales" eran plegados de una forma intrincada, y los modelos que hoy en día son llamados "multiforma", debido a su sencillez y por compartir algunos dobleces entre sí, eran ya populares en Europa durante la segunda mitad del siglo XIX. Estos consisten en la pajarita, el molino de viento, el bote doble y el adivinador o salero (Lister, s.f. a).

El estilo de los modelos de Europa difería significativamente de los de Japón, lo cual refuerza la idea del desarrollo independiente de cada corriente (Hatori, s.f.). Sin embargo, desde la segunda mitad del siglo XIX el país nipón abrió sus fronteras, lo cual llevó a un intercambio de saberes sobre ambos estilos entre los dos territorios. Algunas japonesas salieron del país para aprender sobre industrialización en el occidente, pero también para presentar la cultura oriental, incluyendo su origami (Lister, 2003). Por otro lado, el modelo de educación infantil froebeliano, proveniente de Alemania, llegó a Japón incluyendo sus diferentes actividades de plegado como didácticas de aprendizaje y exploración (Lister, s.f. a). Esto conllevó a la producción, en el país, de papel para origami al estilo occidental: cuadrado, y con una cara de color y otra blanca (Hatori, s.f.).

Continuando con China, donde se originó el papel, sus modelos tradicionales tienen un estilo que difiere del clásico japonés y el europeo. Estos incluyen algunas ofrendas de papel o billetes falsos que se pliegan y queman durante funerales, 
y también, ciertos plegados infantiles. Sin embargo, no se cuenta con información sobre su origen (Lister, 2003).

Ahora, se podría decir que el origami también se originó de forma independiente en Mesoamérica, en el periodo precolombino, aunque de forma más sencilla y este no continuó desarrollándose. Existe evidencia de la producción azteca de un tipo de protopapel, llamado "amate", proveniente de la corteza de diferentes tipos de ficus. Empleaban el amate para el plegado de códices (documentos doblados en zigzag), accesorios y atuendos. Por ejemplo, lo doblaban en forma de abanico o acordeón para usarlo como pendientes para las orejas y como elemento decorativo para la cabeza (Lang, 2014).

Uno de los factores que llevó a la idea diseminada del origami como exclusivamente japonés fue su desarrollo superior, al compararse con el estilo europeo durante la segunda mitad del siglo XIX (Lister, 2003). Otro gran factor fue la difusión de la palabra japonesa "origami", desde la segunda mitad del siglo XX, por parte de la estadounidense Lillian Oppenheimer (Lister, 2003). La palabra "origami" proviene de las raíces japonesas "oru", que significa doblar, y "kami", la cual significa papel (Hatori, s.f.). Los esfuerzos de Lillian Oppenheimer, como fundadora del Origami Center en Estados Unidos y cofundadora de la Sociedad Británica de Origami (BOS por sus siglas en inglés), llevó a la popularización de la palabra a nivel mundial. De hecho, antes de la unificación global del nombre, se usaron también otros en Japón durante periodos históricos diferentes: "orisue", "orikata" y "orimomo" (Hatori, s.f.).

También es muy común la creencia según la cual el origami siempre ha tenido la siguiente serie de reglas: solo está permitido doblar, no cortar, pegar, dibujar, ni colorear, y en una única hoja cuadrada. No obstante, el primer registro conocido de reglas para el origami es relativamente reciente, de 1957, en el libro Paper magic de Robert
Harbin, mago profesional y origamista de origen inglés (Lister, s.f. b). Desde entonces, las reglas del origami se han propagado e incluso han influenciado el origami japonés moderno, a pesar de que su estilo tradicional incluía a menudo cortes.

Respecto a la historia del origami en Suramérica, comisiones de Chile y de Argentina viajaron a Europa a finales del siglo XIX con la intención de aprender sobre su sistema pedagógico. Esto incluía su didáctica basada en el trabajo manual como, por ejemplo, el plegado. De los dos países, fue sobre todo Argentina quien logró instaurar el trabajo manual en su propia educación infantil y, para la década de los 1920, publicó y distribuyó una cartilla dedicada al origami (Sallas, 2012). Sin embargo, el plegado del papel fue retirado de los programas educativos argentinos a mediados del siglo XX (Azcoaga, 2012). Por otro lado, en los años 1930, el origami tuvo mayor divulgación en el país gracias a una entrevista realizada a Vicente Solórzano Sagredo, sobre el plegado del papel y la dedicación y talento de dicho entrevistado, la cual es publicada en el periódico de Buenos Aires "La Prensa”. Solórzano era un odontólogo español radicado en Argentina, cuya afición por este arte lo hacía destacarse (Madueño, 2010).

Ya en lo referente a Colombia, es muy poca la información conocida sobre el surgimiento del origami. El libro de 1961, Testamento del paisa por Agustín Jaramillo Londoño, describe algunas costumbres entre campesinas y aldeanas, desde finales del siglo XIX y hasta mediados del XX. Esto incluye la elaboración de dardos, aviones y helicópteros de papel, así como cálices de envolturas de papel metalizado. Más adelante, en el libro Antología del juguete: Talleres de la infancia de 1968, Euclides Jaramillo Arango comenta sobre algunos juguetes de papel como un bote sencillo y doble, una caja, un corbatín, un adivinador, además de globos, flechas y ringletes. En la misma década, algunos libros y periódicos incluían pasos explicando cómo plegar dichos modelos (jtbm7, 2012). Por tanto, sabemos que en los años 1950, si no es que 
antes, ya existía en Colombia la tradición del plegado del papel entre niñas. Sin embargo, se ignora cómo llegó o se originó tal tradición en el país.

\section{Vida material de los objetos}

El presente ejercicio dialoga con los estudios de la vida material de los objetos (Callén Moreu y López Gómez, 2019; Pérez-Bustos, ChocontáPiraquive, Rincón-Rincón y Sánchez-Aldana, 2019). Tal postura parte del posthumanismo, el cual surge a partir del cuestionamiento del lugar privilegiado de lo humano. En este sentido, rompe con el antropocentrismo, poniendo entre dicho la autonomía e independencia de lo humano frente a lo no-humano, entendiéndolo más bien como resultado de una serie de relaciones y condiciones (Ema López, 2008).

Los estudios de la ciencia y la tecnología (STS por sus siglas en inglés) cuentan con un trayecto significativo en lo que se refiere a la cuestión de los objetos. Law y Singleton (2005) hacen un recorrido por distintas versiones de objetos que el campo ha encontrado y enactado: (1) Objetos como regiones, los objetos del sentido común que ocupan un espacio, en términos euclidianos, al contar con una delimitación física; (2) Objetos como redes, corresponden a los móviles inmutables de Latour y consisten en objetos que simultáneamente movilizan y mantienen su forma funcional y relacional, además de espacial; (3) Objetos fluidos, propuesto por De Laet y Mol, en permanente y lento cambio. Se mantienen como los mismos objetos, en la medida que van cambiando, siempre y cuando no sean cambios acelerados; (4) Objetos fuego, desarrollado por Law y Singleton, los cuales saltan entre distintas formas de manera discontinua y abrupta, dependiendo de tanto presencias como ausencias.

Además de las anteriores cuatro, otras versiones de objetos son posibles (Law y Singleton, 2005). De hecho, fuera de los STS, Henare, Holbraad y Wastell (2007) nos explican algo similar.
Al permitirnos sorprendernos por el qué constituye una $\operatorname{cosa}^{4}$ en un contexto diferente, podremos cuestionar las limitaciones de nuestros supuestos sobre ellas (supuestos ontológicos) y generar una teoría acorde a partir de la cosa misma. Esto, en lugar de basarnos en teorías preexistentes para tratar de explicar dicha cosa. El proceso nos llevaría a conocer muchas más versiones de objetos además del tipo región, redes, fluidos y fuego.

Ahora, mi interés particular respecto a la vida material de los objetos es en cuanto a su vínculo con los afectos. Según Callén Moreu y López Gómez (2019), el concepto de "enredos íntimos" es una herramienta que permite indagar por el proceso que lleva a que un objeto se vuelva importante para alguien, y al cuidado dentro de una relación de interdependencia. Precisamente, el carácter íntimo y cercano de nuestras relaciones con ciertas cosas, en oposición a otras, participan en nuestra constitución material como cuerpos (Sabido Ramos, 2020).

En conexión con lo anterior, la noción de "apego" permite vincular la relacionalidad material con el afecto íntimo compartido entre objetos y aquellas para quienes dichos objetos son importantes. En este contexto, la memoria puede ser de gran importancia, pues los objetos tienen la posibilidad de recrear o mediar ciertos recuerdos, ayudándonos a no olvidar quienes somos y hemos sido, y proyectándolo para otras personas (Callén Moreu y López Gómez, 2019). Al respecto, Sabido Ramos (2020) cita a Francisco Cruces, quien se refiere a los objetos como almacenes de memoria y recuerdos. Adquieren una importancia material y participan directamente, como actantes, sobre nuestro mundo personal.

Siguiendo por la misma línea, también juega un papel el contacto entre cuerpos tanto humanos como no-humanos. Para Ahmed (2014), el contacto es recepción y deja una impresión, una

4"Thing" en el documento fuente.

136 HoJAS Y HABLAS 
marca o trazo. Dicho de otro modo, como cuerpos, nos afectamos mutuamente. El objeto es, de hecho, objeto de mis emociones. Emociones que se relacionan con mis interpretaciones valorativas, pero también, con el contacto entre más cuerpos e historias pasadas de contactos. Puedo ser afectada positiva y también negativamente por un objeto, y la emoción resultante llevará a mi acercamiento o distanciamiento. Ahora, ser objeto de mis emociones no significa que surgen de este ni tampoco de mí, pues a partir de cada contacto afectivo se delimitan los cuerpos de forma diferente, cuerpos que son individuales (yo, lo psíquico) y colectivos (nosotras, lo social), separando así el interior del exterior (Ahmed, 2014).

\section{Detalles metodológicos}

Me basé en la propuesta de investigar con objetos de Callén Moreu y Pérez-Bustos (2020), como un dispositivo de encuentro y sensibilización ante las relaciones y afectos entre cuerpos plegados y los cuerpos humanos que los han cuidado por un tiempo significativo (de aquí en adelante, llamaré a estos otros cuerpos "cuidadoras"). La intención de la propuesta metodológica es procurar las condiciones que permitan captar los afectos y distintas formas de expresión material entre cuerpos.

Comencé con una convocatoria pública en la ciudad de Bogotá. Repartí volantes en reuniones de origami y envié mensajes de correo a diferentes origamistas de la ciudad. Solo una persona me contactó, por lo que cambié de estrategia. Con ayuda de diversos contactos, distribuí un afiche de la convocatoria a través de plataformas sociales y de mensajería instantánea (Instagram, Facebook, WhatsApp, etc.) entre distintas habitantes de la ciudad; no estaba buscando origamistas sino plegados y cuidadoras. De las poco más de 10 que me contactaron, seleccioné cuatro, y mi plegado y yo también participamos, como un componente reflexivo.
Al investigar con objetos, no se pueden ignorar sus ecosistemas. Los objetos forman parte de diferentes redes, al cohabitar con otros, en su espacio doméstico (Callén Moreu y Pérez-Bustos, 2020). Siendo así, visité a cada uno de los plegados en los lugares que habitaban, con la intención de conocer desde adentro: observando y registrando fotográficamente su entorno, y relación con el espacio y otros objetos.

Por otro lado, llevé a cabo entrevistas semiestructuradas junto al plegado y su cuidadora. Estas fueron registradas tanto en video como en fotografía. La imagen captura lo que no dicen las palabras, la articulación entre los cuerpos de los distintos objetos en el espacio y en relación a su cuidadora, a través de los contactos, miradas y gestos compartidos durante la entrevista (Callén Moreu y Pérez-Bustos, 2020).

La entrevista sirvió como un dispositivo elicitador. De acuerdo a Hennion (citado por Callén Moreu y López Gómez, 2019), los dispositivos elicitadores consisten en situaciones que facilitan la afectación sensible de un cuerpo humano ante un objeto en particular; como el encuentro entre un vino y una persona formándose como catadora. El dispositivo nos lleva de pensar sobre el objeto a pensar desde el objeto, como parte de un continuo, enfocándonos en nuestra enmarañada existencia. Entonces, durante las entrevistas con los plegados y sus cuidadoras, rememoraban sus vínculo e historias compartidas $\mathrm{y}$, de ese modo, se sensibilizaban ante el nuevo contacto. Las preguntas fueron en torno a quiénes eran (plegado y cuidadora), las historias y el tiempo compartido, el lugar o los lugares que el plegado ha habitado, sentimientos y emociones que las conectaban, lo que se decían mutuamente, y la relación de la cuidadora con la práctica del origami. En cuanto a mi plegado y yo, una colega nos colaboró como entrevistadora e interlocutora.

$\mathrm{Al}$ investigar con objetos, estos se expresan a través de sus cuerpos humanos durante las en- 
trevistas. Se trata de un acto de ventriloquia que ayuda a que las investigadoras seamos movidas por los objetos. Es una voz que nace de la entrevista misma y los entramados fomentados por el dispositivo elicitador. Pero para que nosotras la aprovechemos y logremos ser movidas, es importante desarrollar la humildad metodológica, reconociendo nuestras limitaciones humanas, y también, siendo atentas y cuidadosas al hacer de las entrevistas dispositivos elicitadores. Por eso, acá procuramos la intimidad en vez de la objetividad (Callén Moreu y Pérez-Bustos, 2020).

\section{Descripción y análisis}

A continuación, presentaré los cinco ejemplos de enredos íntimos cuidadoras-plegados. Todos comparten apegos, afectos, emociones, cuerpos, contactos, memorias, etc., pero de formas lo suficientemente diferentes para presentarnos distintos tipos de relaciones: paternidades, pactos, perdones, retornos e in-materialidades.

\section{Paternidades}

Un origamista pliega su primer modelo complejo a partir de un patrón de plegado ${ }^{5}$ : Dragón ancestral, creado ${ }^{6}$ por Satoshi Kamiya. A través de cada doblez, tanto la hoja como el origamista se iban relacionando materialmente. Se dio el contacto descrito por Ahmed (2014). De hecho, según el cuidador, mientras doblaba le imprimía parte de su propia esencia, pero además él fue un instrumento de la hoja quien sabía desde un comienzo que era un dragón; cosa que ignoraba el origamista-instrumento. Siendo su primera vez, en cualquier momento el origamista podía equivocarse y fallarle a la hoja-dragón, más no fue así. Alcanzó a plegarlo con éxito. Él sintió tanto orgullo por su logro que le dio un nombre al dragón: "Pepito". La emoción surgió del mismo evento compartido. El cuidador delimitó al plegado y lo diferenció de otros mediante el acto de nombrar. A la vez, Pepito también hizo y nombró a un nuevo ser, a través del mismo proceso: a su papá. Establecieron un vínculo de apoyo mutuo entre ambos: el padre desea que Pepito viva más que él, mientras que Pepito, a través del proceso de ser plegado, le comunicó a su padre que no aban-

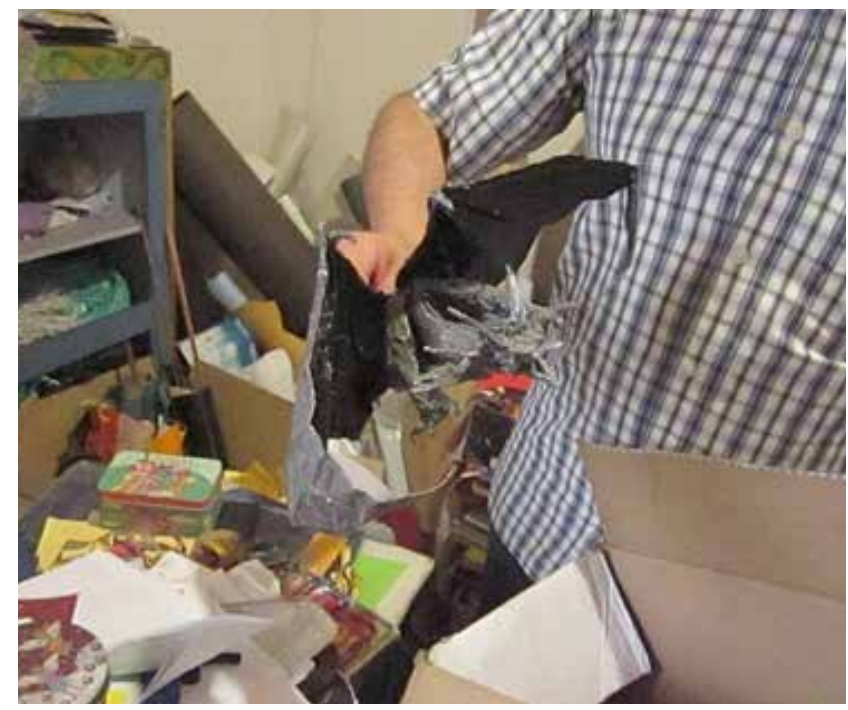

Figura 2. Pepito en el taller y su padre, habiéndolo sacado de su caja.

donara el origami a pesar de todos los obstáculos.

Pactos

Un joven pliega y le obsequia un corazón de origami a su novio en su primera cita al cine. A través del acto, él empezó a formar parte de una red compartida con los dos novios. Se constituyen mutuamente como parte del mismo entramado. Se trató de un plegado de Corazón flor, también llamado Cambio de corazón, creado por Kathleen Weller. Regalar el plegado fue una forma de decirle que, sin importar lo que pasara, siempre

\footnotetext{
${ }^{5}$ Un patrón de plegado (CP por sus siglas en inglés) es un esquema que indica las principales marcas que quedarían sobre el papel si se plegara un modelo y luego se desdoblara por completo. Algunas origamistas saben interpretarlos y así logran plegar los modelos correspondientes.

"Crear" es el término con el que las origamistas se refieren a diseñar un modelo antes inexistente.
} 
tendría un pedazo de su corazón. De esta manera, un pacto se materializó a través del plegado de origami: "Yo te doy una parte de mi corazón. Vos, a cambio, dale el cuidado que necesita". Ellos actualmente no son novios, pero el cuidador mantiene el corazón guardado en su billetera. Él procura cumplir su papel dentro de la red; solo así el plegado seguirá siendo parte de aquel corazón. El cuidador siente que aún le pertenece y por eso lo conserva. Es la emoción que surgió del inter-juego entre los cuerpos, una emoción que lo mantiene cerca del plegado en vez de alejarlo. Tan es así que, es justamente la billetera donde habita el corazón, en contacto con su cuidador y compartiendo un ecosistema con otros objetos importantes como fotos de personas cercanas y la tarjeta de presentación de su naciente proyecto relacionado con mascotas.

A la vez, la billetera como morada era una objeción del corazón-cuidador a mi diseño metodológico; específicamente a la pretensión de visitar todos los plegados en los espacios que habi-

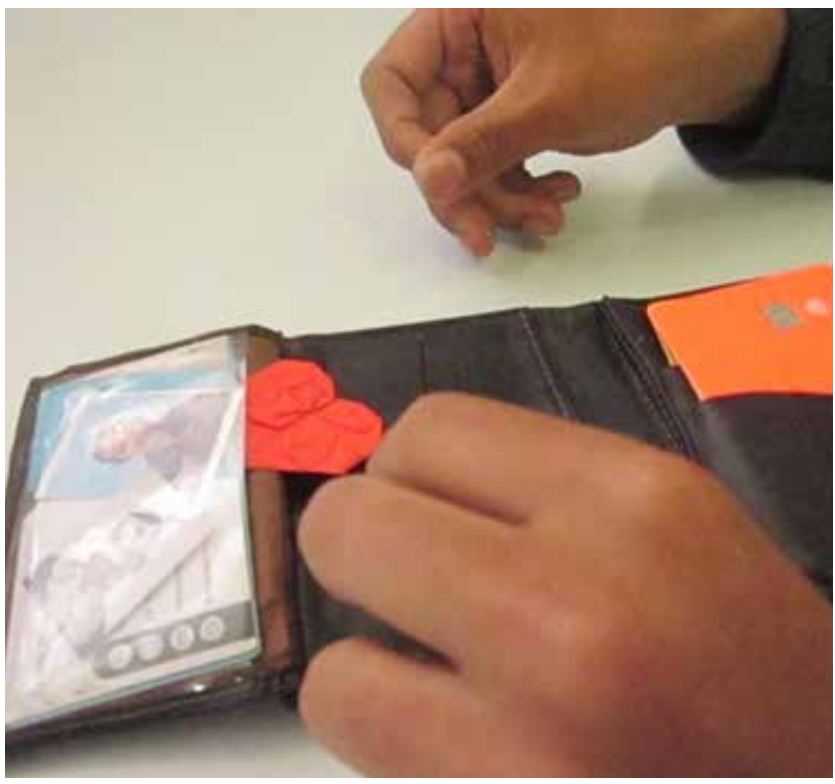

Figura 3. El corazón saliendo de la billetera con la ayuda de su cuidador. tan. Callén Moreu y Pérez-Bustos (2020) advirtieron, como parte de la humildad metodológica, que los objetos pueden llegar a resistir al diseño de nuestros métodos, sorprendiéndonos y haciéndose sentir. Tanto el plegado como el cuidador fueron muy enfáticos en que este habitaba la billetera y no el hogar del cuidador, pues aquel vivía con su mamá y hermana menor, pero ellas no debían acercarse al corazón. El pacto no incluía a ellas dos. De esa manera, delimitan a las participantes y las formas de participación dentro del entramado.

\section{Perdones}

Una madre viaja a Ecuador sin su hijo pequeño para intentar construir un mejor futuro para las dos, pero después de insistir, ella fracasa y se devuelve a Colombia embarazada de su segundo hijo. Las pausas y la entonación de la madre expresan cierta pena durante esta parte de la entrevista. En la emoción participó su interpretación valorativa de los sucesos y también yo, como cuerpo en contacto, mediado por la entrevista. Por aquellos días, su hijo le obsequia el caracol de origami que había hecho. Es un plegado de Caracola en espiral, creada por Toshikazu Kawazaki. En el pasado, su madre le había contado que en México los caracoles eran figuras sagradas del movimiento perpetuo del tiempo. Es decir que, el caracol de origami compartía una red con el hijo, su hermano en gestación, la madre y los caracoles sagrados de México y, a través de dicha red, se afectaban mutuamente. Más cuerpos estaban ahora en contacto, haciendo del caracol de origami la forma corpórea del perdón para la madre ante su proyecto fracasado. Por eso mismo, en la actualidad, el caracol le ayuda a intentar perdonarse por, en palabras de ella, "los errores cometidos con los hijos". Siendo así, el plegado se convierte en un mediador de la memoria de los sucesos pasados, el cual le ofrece la opción de asumirse de una forma diferente. 


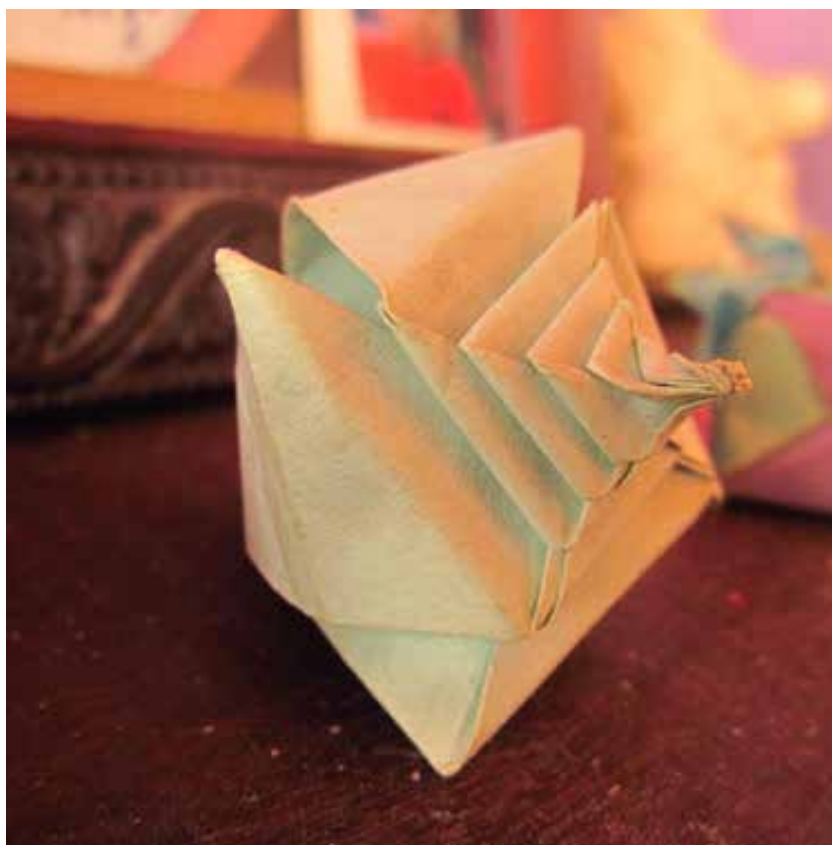

Figura 4. Acercamiento al caracol.

El caracol de origami está ahora junto a la foto de los dos hijos de cuando eran niños y ambas cosas, acompañadas por distintas obras infantiles de arte que ellos hicieron hace ya varios años. Éste es el ecosistema compuesto por el plegado y los otros objetos con los que cohabita. Me explican que aquel ecosistema de fotos, plegados y otras obras está cargado de poder, siendo un altar que ella construyó para que el caracol proteja a sus hijos. No hay que olvidar el vínculo del caracol de origami con los caracoles sagrados de México, además del acto espontáneo y amoroso del hijo, al obsequiarle el plegado a su mamá en el momento más oportuno. Un caracol plegado que protege cuerpos humanos y ayuda a perdonar, nos indica

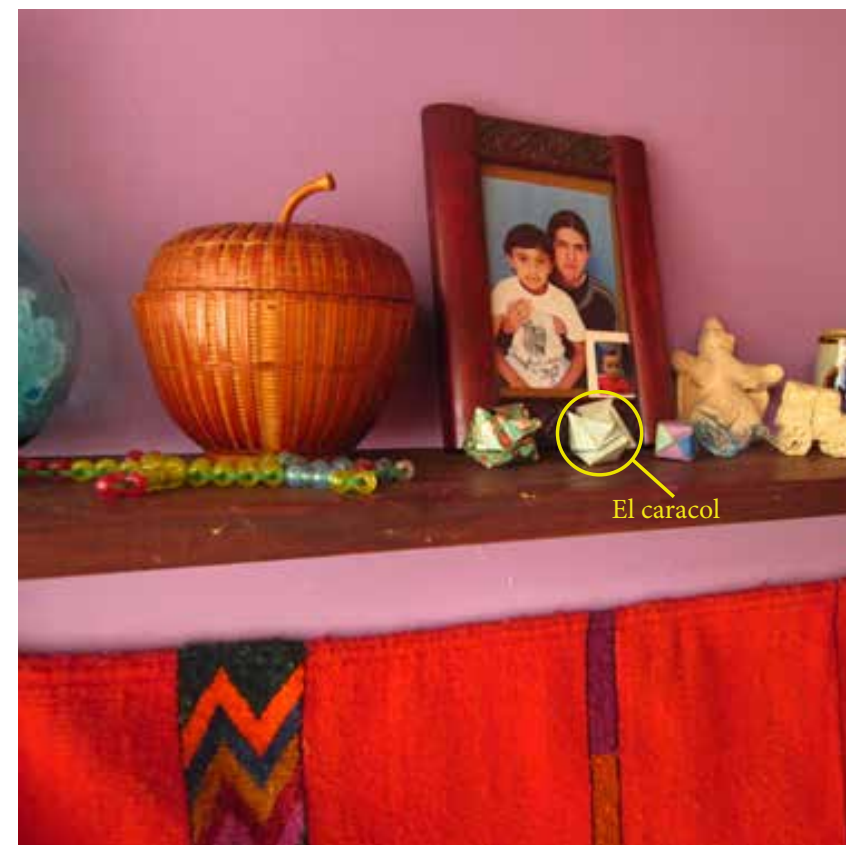

Figura 5. El caracol en el altar desde donde protege a los dos hijos de su cuidadora.

que la relación de cuidado a menudo es recíproca e interdependiente.

\section{Retornos}

Una estudiante pasaba sus tardes en el apartamento de una familia muy cercana a su corazón y el de su mamá. Ella era como otra hija para dicha familia. Una tarde, aquella joven hizo una estrella modular y la dejó en el apartamento; se trató de Estrella Bascetta I también llamada Icosaedro piramidal, creada por Paolo Bascetta. Durante esa noche, se dio el contacto entre la estrella y la hermana mayor. Se afectaron positivamente, configurando a la hermana como cuidadora-dela-estrella, incluso después de abandonar el hogar materno. Desde la mudanza, la estrella cohabita junto a una variedad de artículos de colección, siendo la mayoría regalos por parte de personas que conocen a la cuidadora y a sus gustos. Estos funcionan como índices de memoria, como lo explican Callén Moreu y López Gómez (2019), los cuales proyectan para otras personas quiénes so- 
mos y quiénes hemos sido. Aquel ecosistema, en palabras de la cuidadora, "le genera un valor sentimental a todo el lugar donde está ubicada la estrella”. De esta manera, se comportan como un todo emocional.

La cuidadora me confesó que la estrella es un puente entre épocas, con el cual retorna a esas tardes, a las discusiones entre hermanas y la música, comidas y conversaciones que solía disfrutar en ese entonces. De esta manera, la estrella de origami nos muestra que es un actante que afecta directamente a su cuidadora a través de las memorias y recuerdos que almacena.

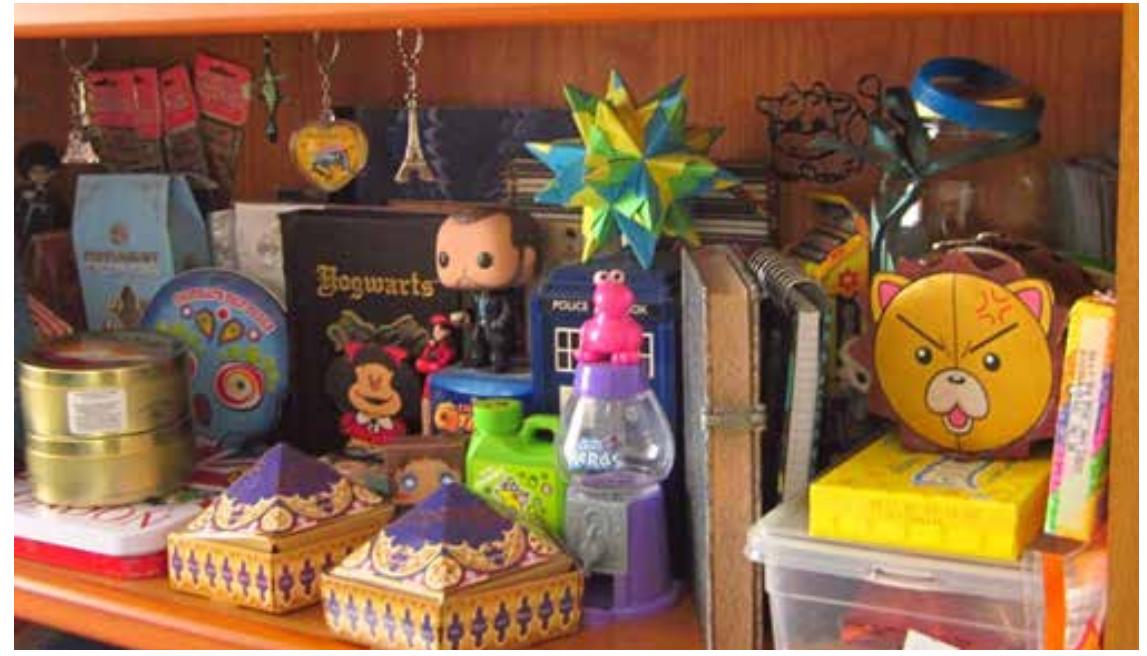

Figura 6. La estrella en su estante junto a diferentes cosas que le han regalado a su cuidadora

\section{In-materialidades}

Una mujer jugó con una serpentina improvisando los dobleces hasta que salió un anillo ${ }^{8}$. Se lo obsequió a su novio origamista. De ese anillo improvisado surgieron diagramas y, así, el anillo se convirtió en un modelo inmaterial que podía ser plegado por distintas manos y en diferentes ocasiones. El cuidador se casó con aquella novia; dejó el anillo en su anterior hogar al mudarse con ella, en el apartamento de sus papás. Hicimos la entrevista en dicho apartamento. Encontramos el anillo dentro de un frasco decorado, en el que su pareja se lo había regalado, al fondo de un estante con juguetes en su antigua habitación.

Durante el desarrollo de la entrevista, él declaro que había olvidado la apariencia del anillo. El anillo-plegado se había vuelto innecesario al existir ahora el anillo-modelo representado en los diagramas. Se trataba de una contradicción que hacía que el cuidador sintiera culpa por inconscientemente rechazar a uno prefiriendo al otro. Esto fue consecuencia de la entrevista, como dispositivo elicitador, la cual le permitió a la versión palpable del anillo imponerse ante su cuidador y, de dicho contacto, surgió la emoción de culpa.

Este anillo de origami parece ser un objeto dinámico y borroso. Así como los objetos fuego de Law y Singleton (2005), el anillo existe a través

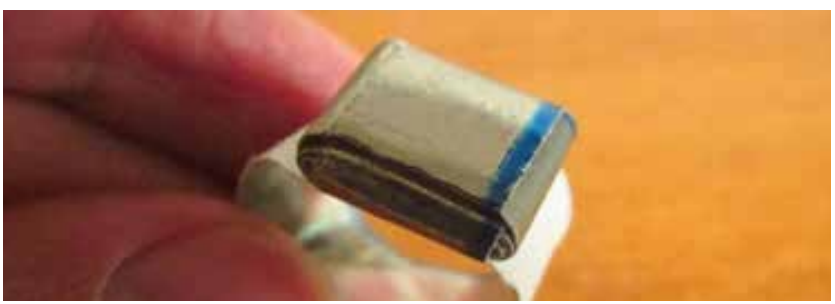

Figura 7. El anillo siendo sostenido por su cuidador.

${ }^{8}$ Omito el nombre del modelo y la creadora para proteger la identidad de las participantes. 


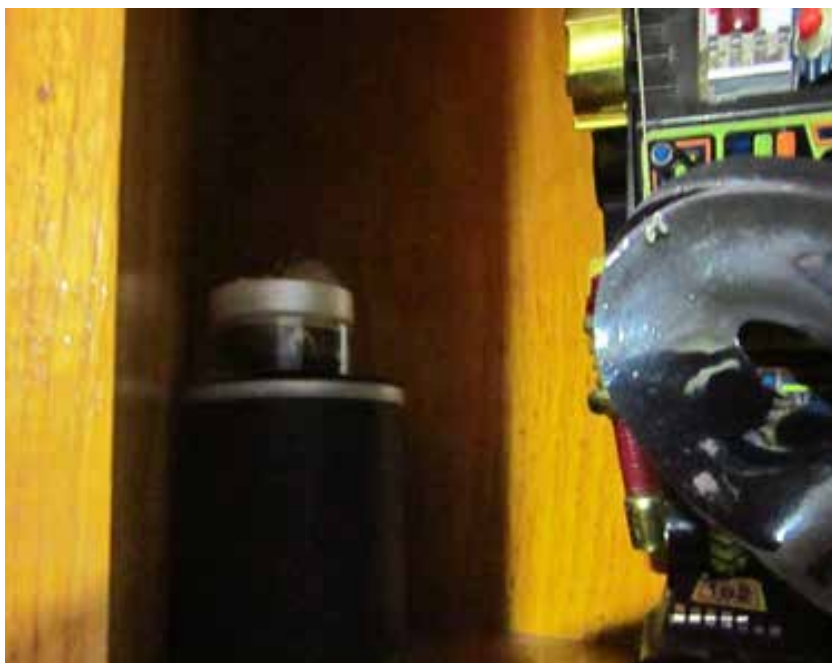

Figura 8. El frasco de vidrio que guardaba al anillo estaba en el fondo de un estante, detrás de unos juguetes.

de presencias y ausencias. Efectivamente, es el cuerpo material que el cuidador tenía en una mano durante la entrevista, pero también es todo anillo potencial que podría salir de cualquier tira apropiada de papel, al seguir los pasos representados en los diagramas. Es así que también existe a través de la ausencia. Es una cuestión de in-materialidad.

\section{Reflexión final}

Presenté cinco ejemplos de cuidado y afecto en las relaciones entre cuerpos humanos y plegados. Aquellos cuerpos plegados que, además de ser hechos a mano, son hechos íntimos dentro de unas relaciones, siguen elaborándose mucho después de terminar de ser doblados. Por tanto, son configuraciones como cosas muy variadas dentro de las relaciones con sus cuidadoras y, a la vez, aquellos también las reconfiguran. Definen así un encuentro particular, en el cual la emocionalidad permite que esta suerte de magia ocurra. Pese a que los cinco eran enredos íntimos entre cuerpos plegados y cuerpos humanos basados en el apego, la relación había sido conformada diferente en cada ejemplo: como paternidades, pactos, perdones, retornos e in-materialidades.

Sin que crea que sea posible documentar todas las configuraciones potenciales, cabe la pregunta de cuáles otras pueden existir entre cuerpos humanos y plegados. Por otro lado, el presente proyecto no me permitió indagar en profundidad sobre los enredos íntimos entre plegados y origamistas. Es posible que la práctica frecuente de plegar sea un factor sobre dichos enredos, por ejemplo, en el caso que la mayoría de plegados efectivamente tiendan a convertirse en bolas de papel. En relación a lo anterior, también hay un gran potencial en indagar por el vínculo en el origami entre creadora, primer plegado y modelo, vínculo al cual le di el apelativo de in-materialidad en el artículo y sugerí su cercanía con los objetos fuego.

\section{Referencias bibliográficas}

Ahmed, S. (2014). The cultural politics of emotion. [Las políticas culturales de la emoción] (2da ed). Escocia: Edinburgh University Press.

Azcoaga, L. (2012). Historia del origami en Argentina. Recuperado de https://www.facebook. com/media/set/?set=a.4008290679475

Callén Moreu, B. y López Gómez, D. (2019). Intimate with your junk! A waste management experiment for a material world. [iÍntima con tu basura! Un experimento de gestión de residuos para un mundo material]. The Sociological Review Monographs, 67(2), 318-339. doi:10.1177/0038026119830318

Callén Moreu, B. y Pérez-Bustos, T. (2020). Metodologías con objetos-objeciones metodológicas. Política y Sociedad, 57(2), 437-458. doi: 10.5209/poso.66452

Da Rivva, D. S. (s.f.). Paper folding in 15th century Europe. [El plegado de papel en la Europa del siglo XV]. Recuperado de: http://www. florilegium.org/files/CRAFTS/Paper-Folding-art.html

Ema López, J. E. (2008) Posthumanismo, materialismo y subjetividad. Política y Sociedad, 45(3), 123-137. Recuperado de: https:// revistas.ucm.es/index.php/POSO/article/ view/POSO0808330123A/21980 
Hatori, K. (s.f.). History of origami. [Historia del origami]. Recuperado de https://origami. ousaan.com/library/historye.html

Henare, A., Holbraad, M. y Wastell, S. (Eds.) (2007). Introduction: Thinking through things. [Introducción: Pensando a través de cosas]. En Thinking through things: Theorising artefacts ethnographically. [Pensando a través de cosas: Teorizando arefactos etnográficamente] (pp. 1-31). Abingdon, Inglaterra: Routledge.

jtbm7 (5 de mayo de 2012). Re: Origami in South America [Comentario en un foro en línea]. [Re: Origami en Suramérica]. Recuperado de https://forum.foldingdidactics.com/ viewtopic.php?t=61

Lang, R. (1987). Because it's there: Wet folding I. [Porque está ahí: Plegado en húmedo I]. British Origami, 125. 20-21.

Lang, R. J. (2014). Editorial: Annals of early origami. [Editorial: Anales del origami temprano]. The Fold, 22. Recuperado de: https:// origamiusa.org/thefold/article/editorial-annals-early-origami

Law, J. y Singleton, V. (2005). Object lessons. [Lecciones de objetos]. Organization, 12(3), 331355. doi.org/10.1177/1350508405051270

Lister, D. (s.f. a). The history of paperfolding: A German perspective. [La historia del plegado de papel: Una perspectiva alemana]. Recuperado de https://britishorigami.info/ lister/german.php

Lister, D. (s.f. b). To glue or not to glue. [Pegar o no pegar]. Recuperado de http://www.britishorigami.info/lister/glue.php

Lister, D. (s.f. c). What is origami? [¿Qué es el origami?]. Recuperado de https://britishorigami.info/lister/what_is_ori.php

Lister, D. (8 de noviembre de 2003). The origin of origami: Is the origin of origami Chinese or Japanese? [El origen del origami: ¿Es el origen del origami chino o japonés?]. Recu- perado de https://britishorigami.info/lister/ origins_of_origami.php

Origami Resource Center (s.f.). History of origami. [Historia del origami]. Recuperado de https://www.origami-resource-center.com/ history-of-origami.html

Madueño, P. (2010). Apuntes sobre el Dr. Vicente Solórzano Sagredo. Plegando al Sur, 1(1). 6-9. Recuperado de http://origamiargentina.org/wp-content/uploads/2020/08/Plegando-al-Sur- ${ }^{\circ} 1 . p d f$

Museo Metropolitano de Arte (s.f.). Models of paper folding (origata tehon) [Modelos del plegado del papel]. Recuperado de https:// www.metmuseum.org/art/collection/ search/78428

Pérez-Bustos, T., Chocontá-Piraquive, A., RincónRincón, C. y Sánchez-Aldana, E. (2019). Hacer-se textil: cuestionando la feminización de los oficios textiles. Tábula Rasa, 32. 249-270. doi:10.25058/20112742.n32.11

Rey, P. J. y Boesel, W. E. (2014). The web, digital prostheses, and augmented subjectivity. [La web, prótesis digitales y subjetividad aumentada]. En Daniel Lee Kleinman y Kelly Moore (Eds.), Routledge handbook of science, technology, and society. [Handbook de Routledge de ciencia, tecnología y sociedad] (pp. 173-188). Abingdon, Inglaterra: Routledge.

Romero Merizalde, D. F. (2015). Del origami a la escultura [Trabajo de grado]. Recuperado de http://www.dspace.uce.edu.ec/ bitstream/25000/4212/1/T-UCE-0002-52. pdf

Sabido Ramos, O. (2020). Sentidos, emociones y artefactos: Abordajes relaciones. Introducción. Digithum, 25. 3-12. Recuperado de https://www.raco.cat/index.php/Digithum/ issue/view/28640/n25-full-issue

Sallas, J. (30 de julio de 2012). Apuntes sobre los orígenes del plegado en Argentina. http:// www.origamiargentina.org/articulos2.htm 